\title{
EFFECTS OF DIFFERENT IMPRESSION METHODS AND HOLDING TIMES ON THE DIMENSIONAL ACCURACY OF ADDITION SILICONES
}

\author{
Shiva Mahboubi', Behnaz Mollai' ${ }^{2}$, Mahdi Rahbar ${ }^{3}$ \\ 'Prosthodontics Department, Dentistry Faculty, Kurdistan University of Medical Sciences, Sanandaj, Iran \\ 2Private Dentist Practitioner, Hamadan, Iran \\ ${ }^{2}$ Restorative and Esthetic Dentistry Department, Dentistry Faculty, Tabriz University of Medical Sciences, Tabriz, Iran
}

\begin{abstract}
INTRODUCTION: Dimensional stability of dies is very important in the success of fixed prosthodontics treatments. Impression methods and holding time are the most important factors affecting dimensional stability of the dies. Овјестіves: This study was performed to investigate the effects of three impression methods and four different dies holding times on the dimensional variations of dies.

MATERIAL AND METHODs: In this in vitro study, 144 gypsum samples were obtained from an in vitro model in the form of prepared pillars of a long bridge - one pyramid dies, with occlusal diameter of $9.43 \mathrm{~mm}$ and height of $12.84 \mathrm{~mm}$, and the other with occlusal diameter of $12.84 \mathrm{~mm}$ and height of $12.80 \mathrm{~mm}$. Two dies distance was $44.20 \mathrm{~mm}$. Forty-eight samples were prepared by one-stage molding, 48 samples by two-stage impression with a spacer on the dies and toothless space, and 48 samples by the two-stage impression and with a spacer on the dies. Each of these three groups was divided into four 12-membered subgroups (1 h, 24 h, 48 h, and one week). Dimensional accuracy of the samples was evaluated by a digital caliper and micrometer. To analyze the data, two-way ANOVA test was used $(p<0.05)$.

RESULTS: The mean of diameter, the height of smaller die, and the distance between the two dies was $9.50 \pm 0.01$, $12.89 \pm 0.02$, and $44.26 \pm 0.01 \mathrm{~mm}$, respectively, in the one-stage impression; $9.46 \pm 0.01,12.87 \pm 0.01$, and 44.22 $\pm 0.01 \mathrm{~mm}$, respectively, in the two-stage impression with spacer on the dies; and $9.46 \pm 0.01,12.87 \pm 0.01$, and $44.22 \pm 0.01 \mathrm{~mm}$, respectively, in the two-stage impression with spacer on the dies and toothless space. There was a significant difference between one-stage and two-stage impression methods $(p<0.05)$, but there was no significant difference between two two-stage impression methods (with different spacer types). Regarding different holding times, no significant difference between them was observed $(p>0.05)$.

Conclusions: The two-stage impression can result in higher dimensional accuracy than the one-stage impression method. Also, when additional silicones are used, the impression can be postponed by one week without significant dimensional changes in the final cast.
\end{abstract}

KEY WORDS: additional silicone, dimensional accuracy, impression method, holding time.

J Stoma 2020; 73, 1: 15-21

DOI: https://doi.org/10.5114/jos.2020.94170

\section{JOURNAL OF STOMATOLOGY CZASOPISMO STOMATOLOGICZNE

ADDRESS FOR CORRESPONDENCE: Dr. Mahdi Rahbar, Restorative and Esthetic Dentistry Department, Dentistry Faculty, Tabriz University of Medical Sciences, Golgasht Street, Tabriz, Iran, e-mail: mahdirhbr@gmail.com

ReCEIVED: 09.08.2019 • ACCEPTED: 16.03.2020 • PUBLISHED: 25.03.2020 


\section{INTRODUCTION}

An impression is the negative form or shape of the original form. The impression is created by placing soft and semi-fluid materials in the mouth and restoring it after being set. Depending on the materials used, the impression may be hard or elastic. Materials that are usually used for casting restorations should be elastic, when taken out of the mouth. Since the restoration must be accurate, the teeth cast from the impression must also be accurate, with no difference. The impression should also be stored carefully before casting. In many cases, the impression shape is changed due to improper maintenance or unnecessary delays between the impression and casting [1].

Despite the advancements in CAD/CAM and the 3D imaging, conventional impression is still widely used. Various factors are effective in the preparation of an accurate impression. Specifically, the conditions of the periodontal membrane of teeth, oral hygiene, location of the finishing line, impression technique, trays used, and precision of the impression material are highly influential [2].

An ideal impression material should not be so hard, so that it can be easily removed from the undercuts. It should also have an elastic recovery property to allow the material to return to its original dimensions once withdrawn from the dental undercuts and prevent it from undergoing a permanent change [3]. It must also have sufficient strength when taken out of the mouth to prevent rupture [4]. Today, there are many types of impression materials, which seem to be reasonably accurate [1]. According to numerous studies, addition silicon or polyvinyl siloxanes are one of the most accurate impression materials in the manufacture of fixed restorations. This material, along with impression materials, has the least variations in casting or recasting [5].

As mentioned above, the impression technique plays a decisive role in the quality of impressions [1]. If the impression methodology is not sufficiently accurate, the impression will undergo dimensional changes, and it will not match correctly at the end of restoration [6]. Common techniques in fixed prosthodontic impression are oneand two-stage methods. In the one-stage method, putty and wash are used simultaneously [7]. The disadvantage of this technique is lack of control over the thickness of the wash [8]. In response, the two-stage impression method is used to overcome the problem of controlling the thickness of wash. In this method, first, the impression is performed by putty, after which, a wash is used in the second stage to record the details [7]. According to ADA no.19, the space provided for the wash material can affect the impression accuracy. If the wash material cannot move freely through the pores of the putty, in the second stage of impressioning, the wash compresses the putty and causes its permanent deformation and irregularities in the impression [4].
Sometimes impressions are send to a laboratory technician at a later time; however, in this case, there is concern about the dimensional variation of impression materials over time [3]. So, this time limit is very important.

\section{OBJECTIVES}

Considering the contradictions in studies, the current paper aims to investigate the effect of different impression methods on the dimensional accuracy of casts and different holding times on the dimensional accuracy of addition silicones. Another purpose of the study was choosing the best method for impression and the best impression time, so that the final restorations have the highest accuracy.

\section{MATERIAL AND METHODS}

The sample size was calculated to be 12 samples in each subgroup, and a total of 144 samples were included with confidence level of $95 \%$ and test power of $90 \%$, as per Sazegara et al. study [7] (taking into account minimum mean of 7.31 , maximum of 7.37 , and standard deviation of 0.04). The 144 samples were prepared to study the effect of different impression methods and holding times on the dimensional accuracy. For each of the main impression groups, 48 samples were prepared based on the impression technique, and these samples were divided into 4 subgroups (each consisting of 12 samples) based on the holding time.

\section{METAL MODEL DEVELOPMENT}

In order to study the dimensional accuracy, the utilized model was in the form of two metal pyramid dies, one with occlusal diameter of $9.39 \mathrm{~mm}$ and height of $12.84 \mathrm{~mm}$, and the other with occlusal diameter of $12.84 \mathrm{~mm}$ and height of $12.80 \mathrm{~mm}$. Each one was placed on a metal pillar with a convergence angle of $6^{\circ}$, $44.20 \mathrm{~mm}$ away from the other (similar to the pillars of a long bridge). Each had a shoulder finishing line of $1 \mathrm{~mm}$ (Figure 1). The obtained measurements in the gypsum samples included diameter and height of smaller die with the two dies distance from at the occlusal part. Finally, the measurements were compared with the main model.

\section{MAKING 5-MM SPACER}

In order to maintain the necessary space for the uniform material and the uniform thickness of the putty at all points, a $5-\mathrm{mm}$ spacer was made with a green acryl (Acropars, Tehran, Iran). A caliper was used to measure the uniform thickness. Equal-sized holes were made in 
the four sides of the spacer, so that when making the acryl tray, the acryl penetrates into the holes and create a stop.

\section{MAKING ACRYLIC SPECIAL TRAY}

To make a special tray, first the 5-mm wax spacer was placed on a metal model and 12 trays were made from green acryl. Tray edges were $10 \mathrm{~mm}$ lower than the horizontal floor of metal model to get enough thickness in the gypsum of samples.

\section{CREATING SPACER FOR TWO-STAGE IMPRESSION TECHNIQUE WITH A SPACER ON DIES AND TOOTHLESS SPACE}

To produce a spacer, 2-mm thick acryl was used on the dies, and the toothless space was elevated. A caliper was used to measure the thickness.

\section{MAKING SPACER FOR TWO-STAGE IMPRESSION TECHNIQUE WITH A SPACER ON THE DIES}

To make this spacer, both dies were covered with a 2-mm thick acryl.

\section{IMPRESSION STAGE}

The main model was subjected to $25^{\circ} \mathrm{C}$ for about $30 \mathrm{~min}$, when the experiment began. The impression material used was the additional silicon (Betasil, Light, Muller Omicron, Germany). The putty was prepared according to the manufacturer's instruction: a base materials of putty was mixed by activator. Also, since the latex material may prevent polymerization of additional silicon, mixing was performed without a latex gloves.

The wash (regular set) was made by the following procedure: after placing the cartridge in the injection gun and putting on the mixer head, pressure was applied on the trigger. In this way, the base and catalyst materials were mixed, and a homogeneous mixture was obtained from the injection gun. The setting time was $5 \mathrm{~min}$, which was considered $10 \mathrm{~min}$ due to in vitro environment.

\section{ONE-STAGE IMPRESSION TECHNIQUE}

Putty and wash were used simultaneously in this technique, where the wash material was prepared concurrently with putty. The putty was then placed inside the tray, while the wash was injected directly around the dies. The experimental model was placed under pressure of 10 psi for $10 \mathrm{~min}$ (Figure 2). Once the im-

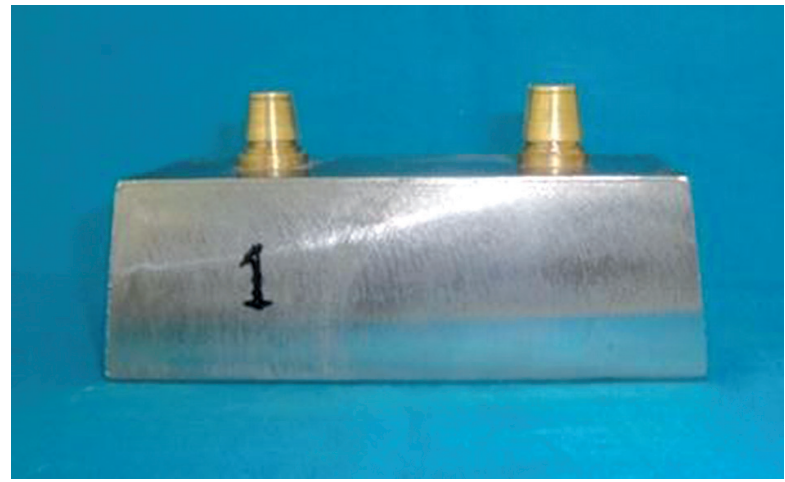

FIGURE 1. Main metal model

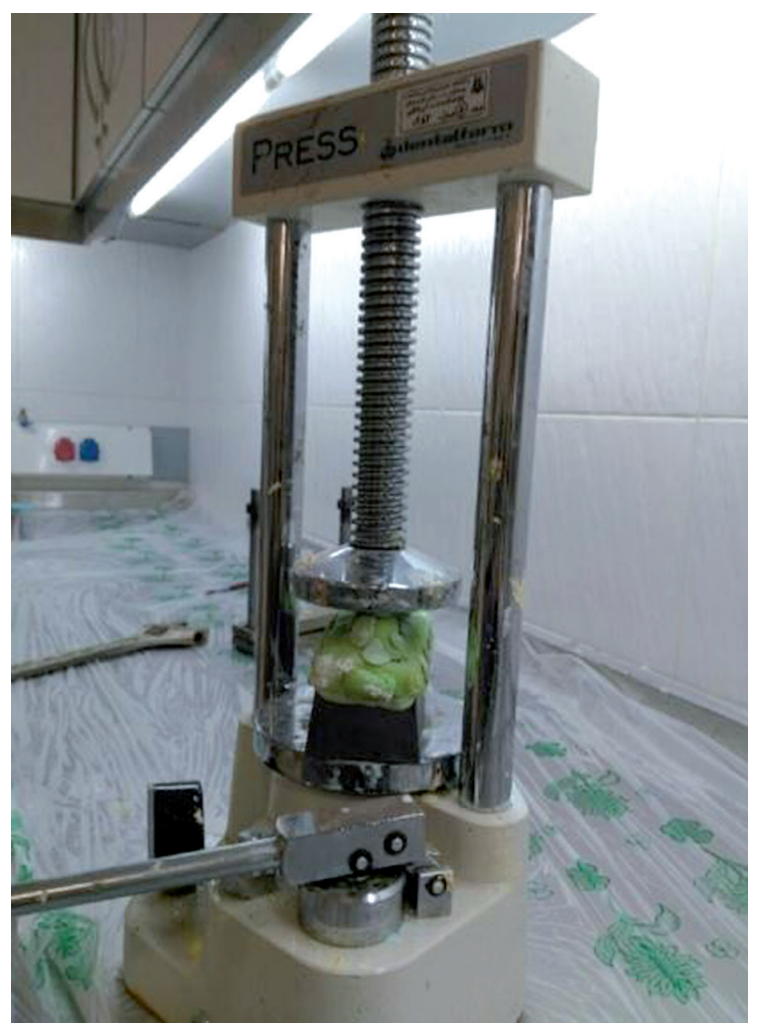

FIGURE 2. In vitro model with tray under pressing machine

pression material was set, the tray was separated from the model.

\section{TWO-STAGE IMPRESSION WITH SPACER ON DIES AND TOOTHLESS SPACE}

In this technique, the pre-prepared spacer was incorporated and the putty was put inside the specific tray, and the model was impressioned in the presence of the spacer. Then, it was placed under pressure for $10 \mathrm{~min}$. After setting, the spacer was removed and the wash was injected around the dies and in the toothless gap. The die was then placed in and underwent a pressure for $10 \mathrm{~min}$. 


\section{TWO-STAGE IMPRESSION WITH SPACER ON DIES}

In this technique, the spacer was placed on the dies. Then, the putty was molded by a specific tray and underwent a pressure for $10 \mathrm{~min}$. Once the putty was set, the spacer was removed from the dies and wash was injected around the dies, with the mold embedded in its place. Again, the mold was pressed for $10 \mathrm{~min}$.

\section{INVESTIGATION OF THE EFFECT OF HOLDING TIME ON DIMENSIONAL ACCURACY OF MOLDS}

To investigate the effect of holding time on the dimensional accuracy, each of the main groups of molds (each group of 48 samples) was divided into 4 subgroups (12 samples) and stored for $1 \mathrm{~h}, 24 \mathrm{~h}, 48 \mathrm{~h}$, and 1 week at $25^{\circ} \mathrm{C}$ and humidity of $30 \%$. Then, the casts were prepared.

\section{CASTING}

The molds were cast with type IV stone (Ariadent, Tahran, Iran). The mixing of stone and water was performed according to the manufacturer's instruction. First,

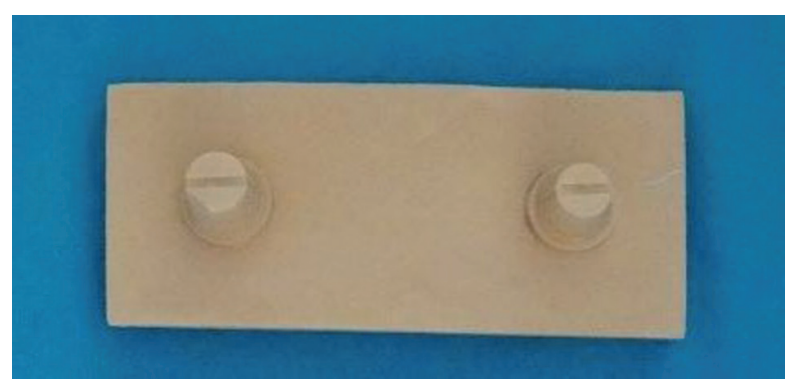

FIGURE 3. A sample of casts removed from the mold

A

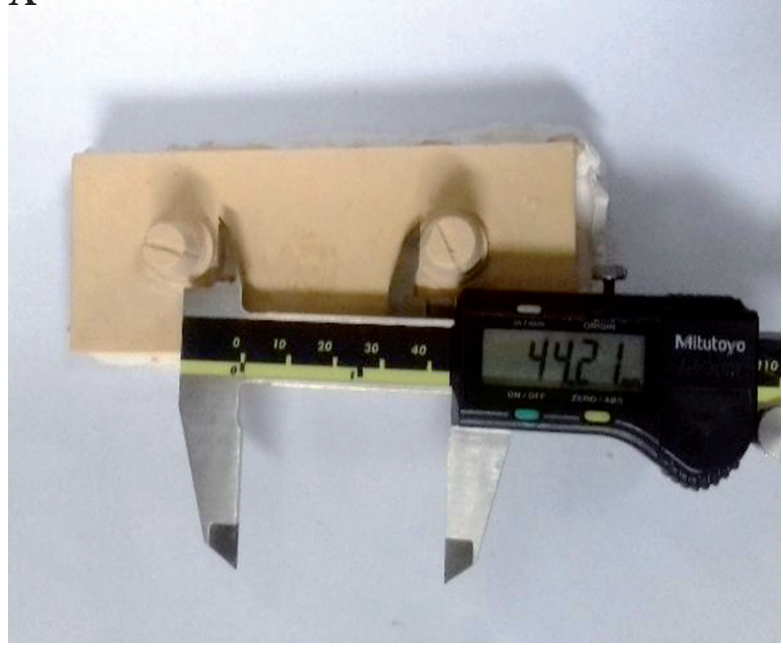

the stone was mixed manually and then mechanically mixed in the vacuum machine for 15 s. Finally, it was poured into the molds under vibration. The molds were removed from the casts $1 \mathrm{~h}$ after casting (Figure 3 ).

\section{MEASURING THE DIMENSIONS OF CASTS}

Measurements of casts included diameter and height of the smaller die and the distance between the two dies. The distance between the two dies was measured by a digital caliper (Mitutoy, Japan), with a measurement accuracy of $0.01 \mathrm{~mm}$. Also, the diameter and height of the smaller die were measured by a digital micrometer (Mitutoy, Japan), with a measurement accuracy of $0.001 \mathrm{~mm}$ (Figure 4).

\section{STATISTICAL ANALYSIS}

Data were analyzed using IBM SPSS Statistics for Windows Software, version 17 (IBM Corp., Armonk, NY, USA) and descriptive statistic with ANOVA test was used. Statistical significance was set at $p<0.05$.

\section{RESULTS}

First, the normal distribution of data was evaluated using the Kolmogorov-Smirnov test. The results showed normal distribution of data $(p>0.05)$. Table 1 reports the results of the mean diameter and height of the smaller die and the distance between the two dies across different impression methods in the gypsum casts.

According to ANOVA, there was a significant difference between various impression methods when examining the three indices (diameter and height of the smaller die as well as the distance between two dies) ( $p$ value $<0.05)$. Based on Scheffe post hoc test, there

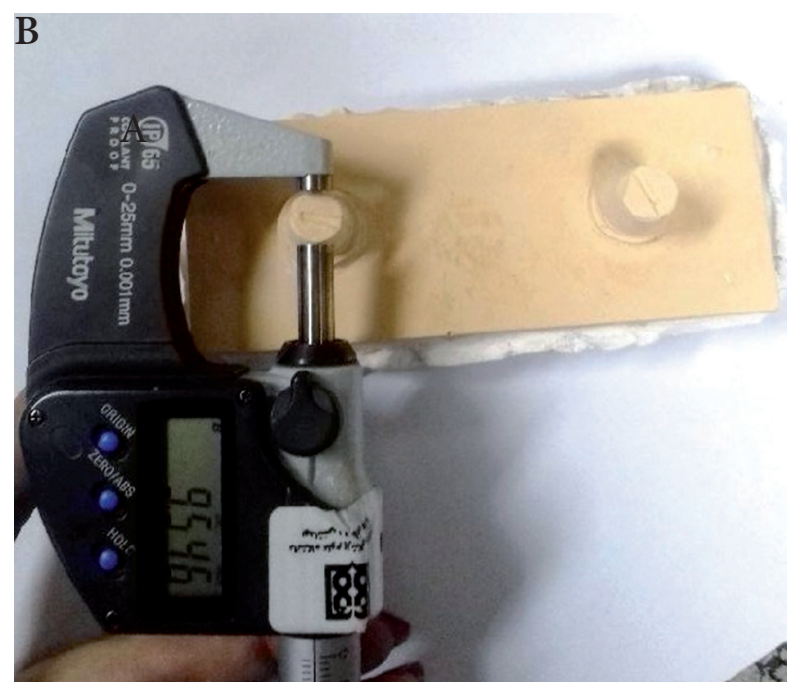

FIGURE 4. A) Measuring the distance between the two dies by the digital caliper. B) Measuring the diameter and height of the smaller die 
TABLE 1. Descriptive statistics of diameter and height of the smaller die and distance between the two dies with different molding methods

\begin{tabular}{|c|c|c|c|}
\hline Indices methods & $\begin{array}{l}\text { Diameter of the smaller die } \\
\text { Mean } \pm \text { standard deviation }\end{array}$ & $\begin{array}{l}\text { Height of the smaller die } \\
\text { Mean } \pm \text { standard deviation }\end{array}$ & $\begin{array}{l}\text { Distance between } \\
\text { the two dies }\end{array}$ \\
\hline One-stage & $0.015^{*} \pm 9.50$ & $0.022^{*} \pm 12.89$ & $0.014^{\mathrm{x}} \pm 44.26$ \\
\hline Two-stage impression with spacer on dies & $0.013^{\#} \pm 9.46$ & $0.019^{\S} \pm 12.87$ & $0.012^{\Delta} \pm 44.22$ \\
\hline $\begin{array}{l}\text { Two-stage impression with spacer on dies } \\
\text { and toothless space }\end{array}$ & $0.011^{\#} \pm 9.45$ & $0.018^{\S} \pm 12.85$ & $0.015^{\Delta} \pm 44.22$ \\
\hline
\end{tabular}

Same symbols in every column mean no statistically significant differences between the groups $(p>0.05)$, and different symbols indicate statistically significant differences between the groups $(p<0.05)$.

TABLE 2. Descriptive statistics of diameter and height of the smaller die and distance between the two dies at different holding times

\begin{tabular}{|c|c|c|c|}
\hline Indices times & $\begin{array}{l}\text { Diameter of the smaller die } \\
\text { Mean } \pm \text { standard deviation }\end{array}$ & $\begin{array}{l}\text { Height of the smaller die } \\
\text { Mean } \pm \text { standard deviation }\end{array}$ & Distance between the two dies \\
\hline $1 \mathrm{hr}$ & $0.025^{*} \pm 9.477$ & $0.022^{\triangle} \pm 12.877$ & $0.022^{*} \pm 44.24$ \\
\hline $24 \mathrm{hr}$ & $0.025^{*} \pm 9.474$ & $0.021^{\Delta} \pm 12.874$ & $0.021^{*} \pm 44.23$ \\
\hline $48 \mathrm{hr}$ & $0.022^{*} \pm 9.476$ & $0.022^{\Delta} \pm 12.875$ & $0.022^{*} \pm 44.24$ \\
\hline 1 week & $0.021^{*} \pm 9.472$ & $0.076^{\perp} \pm 12.876$ & $0.023^{*} \pm 44.23$ \\
\hline
\end{tabular}

Same symbols in every column mean no statistically significant differences between the groups $(p>0.05)$.

was a significant difference between one-stage and twostage methods across all three dimensions. However, there was no significant difference between the twostage method with spacer on the die, and with spacer on the die and the toothless gap ( $p$ value $>0.05$ ).

Mean diameter and height of the smaller die and distance between the two dies at different holding times of the molds are presented in Table 2. According to ANOVA, there was no significant difference between different holding times of dies templates for any dimension $(p>0.05)$.

According to the comparison of mean numbers obtained from the casts with the dimensions of main model, the accuracy of two-stage impression method was higher than that of one-stage one. This means that the measurements obtained from two-stage impression methods were closer to dimensions of the original model.

\section{DISCUSSION}

In fixed prosthodontics treatments, it is essential to provide an accurate mold to obtain appropriate restorations. An impression technique plays a decisive role in the quality of molds obtained. If the impression technique is not as accurate as required, the mold will undergo dimensional changes, and the restoration will not match appropriately [6].

In this study, the accuracy of three different impression techniques was studied (one stage, two-stage with spacer on the dies, and two-stage with spacer on the dies and toothless gap). Four different holding times were investigated for the mold ( $1 \mathrm{~h}, 24 \mathrm{~h}, 48 \mathrm{~h}$, and one week) before casting. The dimensions measured included the dimension and height of the smaller die and the distance between the two dies. The results revealed a significant difference between one- and two-stage methods, where the accuracy of two-stage impression was higher than that of one-stage. However, no significant difference was found between the two-stage methods with various spacers. According to numerous studies, the addition silicon and polyvinyl siloxanes are the most accurate impression materials in fixed restorations regarding holding time of a mold. These materials have a minimum variations when a delay occurs in casting or recasting [9-11]. In the study, statistical analysis showed no significant difference between various holding times. Different impression methods had no mutual effects on each other either. In a similar study by Caputi et al. comparing the dimensional accuracy of one-stage, two-stage with a $2 \mathrm{~mm}$ spacer, and two-stage injection impression method, it was concluded that two-stage method with spacer and two-stage injection method were more accurate than one-stage method, which is consistent with our present study [12]. In another study, the dimensional accuracy of three different impression methods was investigated, which used polyvinyl siloxane: one-stage method, two-stage method with 2 -mm relief in the putty, and two-stage method with polyethylene spacer. They finally concluded that this two-stage method with 2-mm relief in the putty was the most accurate [8]. The results were somehow similar to findings of the present study.

In another similar study, comparison of the effect of three impression methods on the dimensional variation of dies indicated that impression techniques had 
an impact on the dimensional accuracy, where two-stage impression technique with a $2-\mathrm{mm}$ spacer was more accurate than two-stage method without spacer and onestage method [13]. Although the material used in this study was a condensation silicone impression (Speedex), the method of spacing and its volume (wash thickness) were similar to our study. The study of two two-stage method with a foil spacer and a putty cutout method suggested that the two-stage impression with foil spacer (61.4 to $64.4 \mathrm{~mm}$ ) was more accurate than the cut out two-stage method (55.4 to $61.4 \mathrm{~mm}$ ) [14]. The reason for the accuracy of two-stage method with a foil spacer was the uniform thickness of wash, which is somewhat similar to that of our study.

Comparison of the dimensional accuracy of onestage method with the putty-wash two-stage method with 5 types of addition silicone, in which a metal tray was used demonstrates that the dimensional accuracy was more influenced by the type of impression material than by the impression method. Further, only in one of the measured dimensions, the one-stage method had a higher accuracy compared to its two-stage counterpart [15]. The results of this study contradict our findings. The reason for this discrepancy can be the type of impression material, impression technique, and in vitro conditions.

Rathee et al. investigated the dimensional accuracy of addition silicon in impression with various spacers. Two spacers were used for molding, one only covering the die and the other covering the die and the toothless gap. The authors concluded that the impression method, in which the spacer covered the die and the toothless gap simultaneously was more accurate [16]. The results of this study have not been consistent with ours. The different impression material brand (Dentsply) and in vitro conditions can be the reasons for inconsistency. In another study, the effect of three impression methods was investigated on the dimensional accuracy of die. In this study, the in vitro model consisted of three pillars and a type of addition silicon called Affinis used as impression material. Three techniques were used to prepare the molds: one-stage, two-stage without spacer, and two-stage with spacer. The final casts were measured by a profilometer microscope and a micrometer. The authors observed that impression methods did not affect dimensional variations [7]. The results of this study have not been consistent with our findings. The difference between the impression material brand and different in vitro conditions could be the reasons for inconsistency.

Idris et al. conducted a comparative study on the effects of one- and two-stage impression methods on the dimensional accuracy of die. For each method, 15 molds were prepared and cast. They concluded that variations were clinically negligible and that differences between various impression methods were not significant [17]. The results of this study have not been consistent with the present study outcomes. In this study, a special metal tray was used, which could minimize mold variations due to hardness. However, in our study, a specific acrylic tray was used.

A similar study was conducted by Mosharraf et al. on the effect of holding time on dimensional variations of polyvinyl siloxane impression material. In this study, an in vitro model consisting of three pillars was prepared. The molds were divided into four 10-member groups, with each cast at $1,4,24$, and $48 \mathrm{~h}$ post-molding. Statistical analysis did not show any significant difference between these four groups [18]. However, the results have been compatible with our findings.

Thongthammachat et al. studied the dimensional accuracy of casts for different types of tray, impression materials, and holing times. In this study, four different types of materials were used to make the tray, two types of materials for molding, and four different holding times for molds ( $30 \mathrm{~min}, 6 \mathrm{~h}, 24 \mathrm{~h}$, and 30 days). The two materials used for impression included polyether and addition silicon. After measuring the dimensions, they concluded that the addition silicon did not undergo dimensional changes until 30 days after impression (change is negligible) [19]. The result of this study was consistent with our observations. Levartovsky et al. investigated the effect of one-stage and two-stage impressions using the addition silicon as well as different holding times of molds. They prepared 15 casts in each method, where the holding durations of the molds were $0.5,2,24,48,72,96$, and $120 \mathrm{~h}$. Measurements were done by a microscope. They concluded that the two-stage impression method was more accurate than the single-stage one. In case of the one-stage method, the molds should have been casted for $2 \mathrm{~h}$, but in a two-step method, the casts would not experience

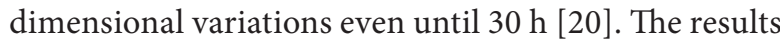
of this study have not been consistent with our study in terms of the mutual effects of time and the impression method. However, they have been similar concerning the accuracies. The differences can be due to the difference in the brand of addition silicone (President) and in vitro conditions.

In another study, the effect of holding time was investigated on the viscoelastic properties of elastomeric impression materials. Six types of impression materials from different brands (five types of silicones [compression or addition] and one type of polyether) were used. After being stored for $30 \mathrm{~min}$ to 2 weeks, they were tested both statically and dynamically. The results revealed that the holding time had a significant effect on their viscoelastic properties. All of these impression materials exhibited linear viscoelastic behavior and they underwent permanent deformation after $50 \mathrm{~h} \mathrm{[3].} \mathrm{The} \mathrm{re-}$ sults of this study were dissimilar with our results. The tests in this study were performed on molds, while in the present study, they were performed on die casts. Dimensional changes of stone (stone brand, powder to water ratio, in vitro storage conditions) could also affect the results. 
Considering the importance of obtaining an accurate cast in the success of prosthodontics, it is recommended that further studies are performed to investigate the effects of other impression techniques, different holding times of mold, and various prosthodontic treatments (fixed prosthodontic, partial prosthodontic, implant). It is also recommended to study other factors affecting the success of fixed prosthodontics, such as waxing up, casting, and porcelain.

\section{CONCLUSIONS}

Considering the limitation and conditions of the study, two-stage impression could result in casts with higher dimensional accuracy than their one-stage counterparts. Also, in case of using addition silicones, impression could be postponed by one week without significant dimensional changes in the final cast.

\section{CONFLICT OF INTEREST}

The authors declare no potential conflicts of interest with respect to the research, authorship, and/or publication of this article.

\section{References}

1. Mousavi S, Rahbar M, Rostamzadeh F, Jafari K, Hekmatfar S. Dimensional stability of casts derived from three types of alginate at different times after impression. Pesqui Bras Odontopedia Clin Integr 2019; 19: 4137.

2. Stober T, Johnson GH, Schmitter M. Accuracy of the newly formulated vinyl siloxanether elastomeric impression material. J Prosthet Dent 2010; 103: 228-239.

3. Papadogiannis D, Lakes R, Palaghias G, Papadogiannis Y. Effect of storage time on the viscoelastic properties of elastomeric impression materials. J Prosthodont Res 2012; 56: 11-18.

4. Sakaguchi RL, Powers JM. Craig's restorative dental materials e-book. Elsevier Health Sciences, 2012.

5. Johnson GH, Craig RG. Accuracy of four types of rubber impression materials compared with time of pour and a repeat pour of models. J Prosthet Dent 1985; 53: 484-490.

6. Small B. Important factors for accurate impressions. Gen Dent 2001; 49: 260-263.

7. Sazegara H, Nahidi R. Effect of impression tray on accuracy of implant position transferring in close tray method using polyvinyl siloxane. Journal of Dental School Shahid Beheshti University of Medical Science 2009; 26: 346-354.

8. Nissan J, Laufer BZ, Brosh T, Assif D, Maurice T. Accuracy of three polyvinyl siloxane putty-wash impression techniques. J Prosthet Dent 2000; 83: 161-165.

9. Willis CL, Handlin Jr DL, Trenor SR, Mather BD. Method for varying the transport properties of a film cast from a sulfonated copolymer. Google Patents 2011.

10. Surapaneni H, Yalamanchili PS, Yalavarthy RS, Attili S. Polyviny siloxanes in dentistry: an overview. Trends Biomater Artificial Organs 2013; 27: 115-123.

11. Mehta R, Dahiya A, Mahesh G, et al. Influence of delayed pours of addition silicone impressions on the dimensional accuracy of casts. J Oral Health Comm Dent 2014; 8: 148-153.

12. Caputi S, Varvara G. Dimensional accuracy of resultant casts made by a monophase, one-step and two-step, and a novel two- step putty/light-body impression technique: an in vitro study. J Prosthet Dent 2008; 99: 274-281.

13. Mahdavy-Izady Z, Jalalian E, Neshandar M, Arazm-Sa A, Meyhami A. Die dimension variation in three impression taking methods. Journal of Islamic Dental Association of Iran 2009; 20: 286-291.

14. Mann K, Davids A, Range U, Richter G, Boening K, Reitemeier B. Experimental study on the use of spacer foils in two-step putty and wash impression procedures using silicone impression materials. J Prosthet Dent 2015; 113: 316-322.

15. Hung SH, Purk JH, Tira DE, Eick JD. Accuracy of one-step versus two-step putty wash addition silicone impression technique. J Prosthet Dent 1992; 67: 583-589.

16. Rathee S, Eswaran B, Eswaran M, Prabhu R, Geetha K, Krishna G A comparison of dimensional accuracy of addition silicone of different consistencies with two different spacer designs - in-vitro study. J Clin Diagn Res 2014; 8: ZC38.

17. Idris B, Houston F, Claffey N. Comparison of the dimensional accuracy of one-and two-step techniques with the use of putty/wash addition silicone impression materials. J Prosthet Dent 1995; 74: 535-541.

18. Mosharraf R, Nosouhian S, Salehi M. Effect of storage time on dimensional stability of Extended-pour irreversible hydrocolloid impression material. J Isfahan Dent Sch 2011; 7: 246-255.

19. Thongthammachat S, Moore BK, Barco MT, Hovijitra S, Brown DT, Andres CJ. Dimensional accuracy of dental casts: influence of tray material, impression material, and time. J Prosthodont 2002; 11: 98-108.

20. Levartovsky S, Zalis M, Pilo R, Harel N, Ganor Y, Brosh T. The effect of one-step vs. two-step impression techniques on long-term accuracy and dimensional stability when the finish line is within the gingival sulcular area. J Prosthodont 2014; 23: 124-133. 